\title{
Wellens' Syndrome: A Bad Omen
}

\author{
Madalena Coutinho Cruz ${ }^{\mathrm{a}}$ Isabel Luiz $^{\mathrm{b}}$ Lurdes Ferreira ${ }^{\mathrm{a}}$ Rui Cruz Ferreira ${ }^{\mathrm{a}}$ \\ ${ }^{a}$ Cardiology Department, Hospital de Santa Marta, and b Internal Medicine Department, Hospital de São José, \\ Centro Hospitalar de Lisboa Central, Lisbon, Portugal
}

\section{Established Facts}

- Wellens' syndrome is a relatively common clinical entity with clear diagnostic criteria; however, it is frequently missed.

- The lack of recognition of this syndrome has clear prognostic implications since the majority of patients who are maintained on medical therapy progress to an anterior myocardial infarction.

\section{Novel Insights}

- The spectrum of Wellens' syndrome is very wide and some patients will fare well with medical therapy while others will need urgent revascularization.

- There are no established criteria to distinguish these two groups of patients and a high clinical suspicion of this syndrome remains the best weapon against its consequences.

\section{Keywords}

Left anterior descending artery · Acute coronary syndrome . Unstable angina $\cdot$ T wave

\begin{abstract}
Wellens' syndrome is characterized by an electrocardiographic pattern of T-wave changes associated with a critical stenosis of the left anterior descending artery (LAD), which progresses to an extensive anterior myocardial infarction in the majority of cases. For this reason, its recognition and early treatment are extremely important. We report 2 cases of Wellens' syndrome: an 83-year-old male presenting with illcharacterized chest pain, biphasic T waves in V1-V3 during
\end{abstract}

an asymptomatic period, negative cardiac biomarkers, and a $64 \%$ stenosis in LAD with a fractional flow reserve of 0.96 who fared well on medical therapy, and an 67-year-old male with typical angina pain, biphasic T waves in V2-V4 during asymptomatic periods, anterior ST-segment elevation at 2 min of effort during an exercise stress test, positive highsensitivity cardiac troponin, and an $80 \%$ stenosis in the proximal LAD who was submitted to percutaneous coronary intervention which rendered him asymptomatic. The spectrum of Wellens' syndrome is very wide, and knowledge and high clinical suspicion for its diagnosis, especially in its rarest presentation of biphasic T waves, is key to avoiding catastrophic consequences.

(c) 2017 S. Karger AG, Basel

\section{KARGER}

(c) 2017 S. Karger AG, Basel

E-Mail karger@karger.com

www.karger.com/crd
Madalena Coutinho Cruz

Serviço de Cardiologia, Hospital de Santa Marta

Centro Hospitalar de Lisboa Central, Rua de Santa Marta

PT-1169-1024 Lisbon (Portugal)

E-Mail madalena.cruz@chlc.min-saude.pt 


\section{Introduction}

The first report of Wellens' syndrome was published in 1982 by Wellens and colleagues, who described an electrocardiographic pattern seen in patients admitted for unstable angina, characterized by $\mathrm{T}$-wave changes in the precordial leads, that was related to a stenosis of the proximal left anterior descending artery (LAD) [1]. The criteria for diagnosing this syndrome are: a prior history of chest pain, no elevation of cardiac markers or elevation up to 2 times the upper limit of the normal reference value, an isoelectric or slightly elevated $(<1 \mathrm{~mm})$ ST segment, no precordial $\mathrm{Q}$ waves, no loss of precordial $\mathrm{R}$ waves, and symmetrically inverted or, less commonly, biphasic T waves in the precordial leads [2]. Early recognition of these patients is extremely important since the majority of patients will progress to an extensive anterior myocardial infarction (MI) in a matter of days to weeks if maintained on conservative therapy [1]. The performance of a stress test, as is frequently required when evaluating patients with unstable angina, is contraindicated in this kind of patient as it can trigger an anterior MI and result in death [2].

\section{Case Reports}

\section{Case Report 1}

An 83-year-old male with hypertensive heart disease, congestive heart failure, carotid atherosclerosis, and incipient dementia presented to the emergency department with ill-characterized chest pain and dyspnea that had been present intermittently for a few weeks and appeared with increasingly low effort. He had increased jugular venous pressure, inspiratory crackles in the lung bases, and peripheral edema. An electrocardiogram (ECG) revealed sinus rhythm and left ventricular hypertrophy with ST changes in V1-V3 and asymmetric T-wave inversion in the lateral and inferior leads, which was fairly equal to the previous exam (Fig. 1a). The cardiac troponin I (cTnI) level was $0.12 \mathrm{ng} / \mathrm{mL}$ (normal value $<0.032 \mathrm{ng} / \mathrm{mL}$ ) and the brain natriuretic peptide (BNP) level was $271 \mathrm{pg} / \mathrm{mL}$ (normal value $<100 \mathrm{pg} / \mathrm{mL}$ ).

He was admitted with a diagnosis of congestive heart failure and improved with diuretic therapy. Four days after the admission, bradycardia ( 46 beats/min) was noted in the physical examination, which prompted the requisition of an ECG. It revealed biphasic T waves in $\mathrm{V} 1-\mathrm{V} 3$ and pseudonormalization of the inverted $\mathrm{T}$ waves (Fig. 1b). The cTnI level was normal and the patient was pain free. A transthoracic echocardiogram displayed anterior septum hypokinesis, biauricular, and left ventricle dilation, and a left ventricle ejection fraction of $47 \%$. A coronariography showed a $64 \%$ stenosis in the proximal LAD by quantitative measurement with a fractional flow reserve of 0.96 . The patient was medicated according to the diagnosis of acute coronary syndrome and fared well with medical therapy.

Wellens' Syndrome: A Bad Omen

\section{Case Report 2}

A 67-year-old male with no previously known diseases complained of episodes of oppressive chest pain with irradiation to both arms for a few weeks, which appeared initially during effort but progressed to resting pain, with increasing frequency and intensity. The attending physician requested an exercise stress test that showed a biphasic T wave in V2-V4 at baseline and an STsegment elevation of $7 \mathrm{~mm}$ in V2-V4 at 2 min of effort with complete resolution after $10 \mathrm{~min}$ of rest (Fig. 2a). The patient was rushed to the emergency department and an ECG confirmed the biphasic $\mathrm{T}$ waves in $\mathrm{V} 2-\mathrm{V} 4$ during an asymptomatic period. The high-sensitivity cTnI level was $290.6 \mathrm{pg} / \mathrm{mL}$ (normal value $<34.2$ $\mathrm{pg} / \mathrm{mL}$ ). A coronariography revealed an $80 \%$ obstruction of the proximal LAD that was treated with a drug-eluted stent. Optimal coherence tomography was used to exclude an ostial lesion. The patient remained asymptomatic after the procedure and optimized medical therapy.

\section{Discussion}

Wellens' syndrome is not a rare event since $14-18 \%$ of all patients with unstable angina in the original studies fulfilled the criteria. The more common of the 2 patterns of $\mathrm{T}$-wave changes were deep symmetrically inverted $\mathrm{T}$ waves, which were present in approximately three fourths of the patients. These kinds of T-wave changes are mostly recognized as a marker for ischemia and the patients are managed accordingly. On the other hand, the rarest pattern of biphasic $\mathrm{T}$ waves is frequently interpreted as nonspecific repolarization changes and sometimes ignored, which can have dire consequences for the patient [1-3]. T-wave inversions are a common finding and have a broad differential diagnosis, but if one takes the syndrome's criteria into account or if biphasic $\mathrm{T}$ waves are present, the scenario is quite specific and should not be missed [4]. In both cases, the biphasic $\mathrm{T}$ waves were most prominent in $\mathrm{V} 2-\mathrm{V} 3$, but they were also present in V1 (in the first case) and V4 (in the second case), which is in accordance with the original study where all patients displayed $\mathrm{T}$-wave changes in V2-V3, the majority also in $\mathrm{V} 1-\mathrm{V} 4$, and a few in V5-V6 [1].

With regard to the first case presented, the patient suffered from incipient dementia and had difficulty recalling and characterizing events, which influenced the fact that the diagnosis made in the emergency department was congestive heart failure and the cTnI rise was interpreted in this context. In hindsight, the pain was probably cardiac in origin and the lack of ischemic ECG changes should not dismiss the diagnosis of acute coronary syndrome, because it is common in this group of patients during symptomatic periods [3]. Besides, the 


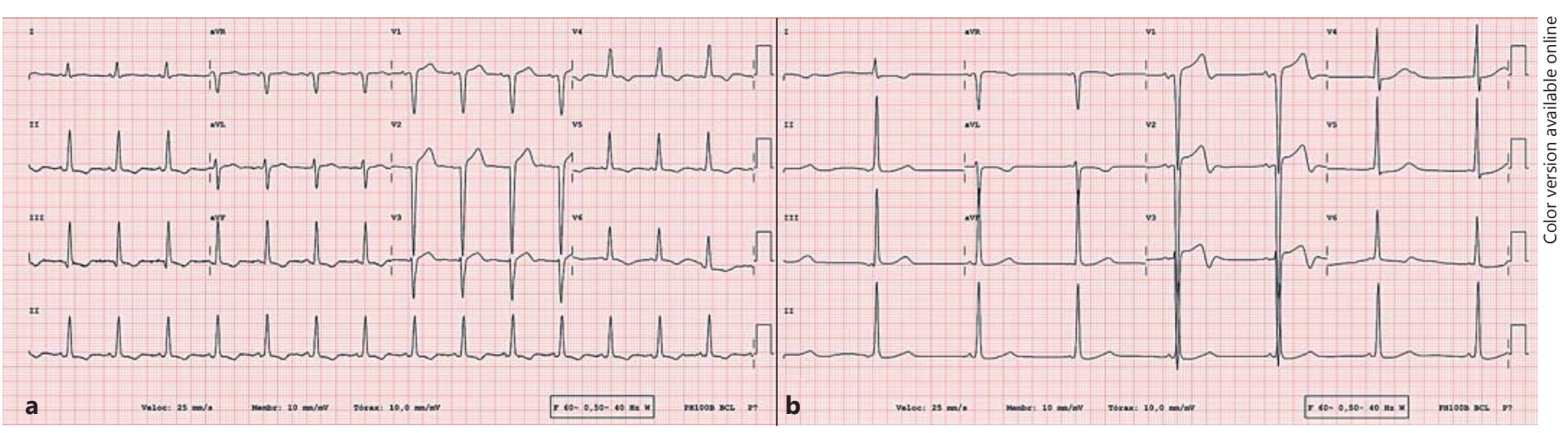

Fig. 1. a Initial electrocardiogram tracing with left ventricular hypertrophy with ST-changes in V1-V3 and asymmetric T-wave inversion in the lateral and inferior leads. b Electrocardiogram with biphasic T waves in V1-V3 and pseudonormalization of inverted $\mathrm{T}$ waves.

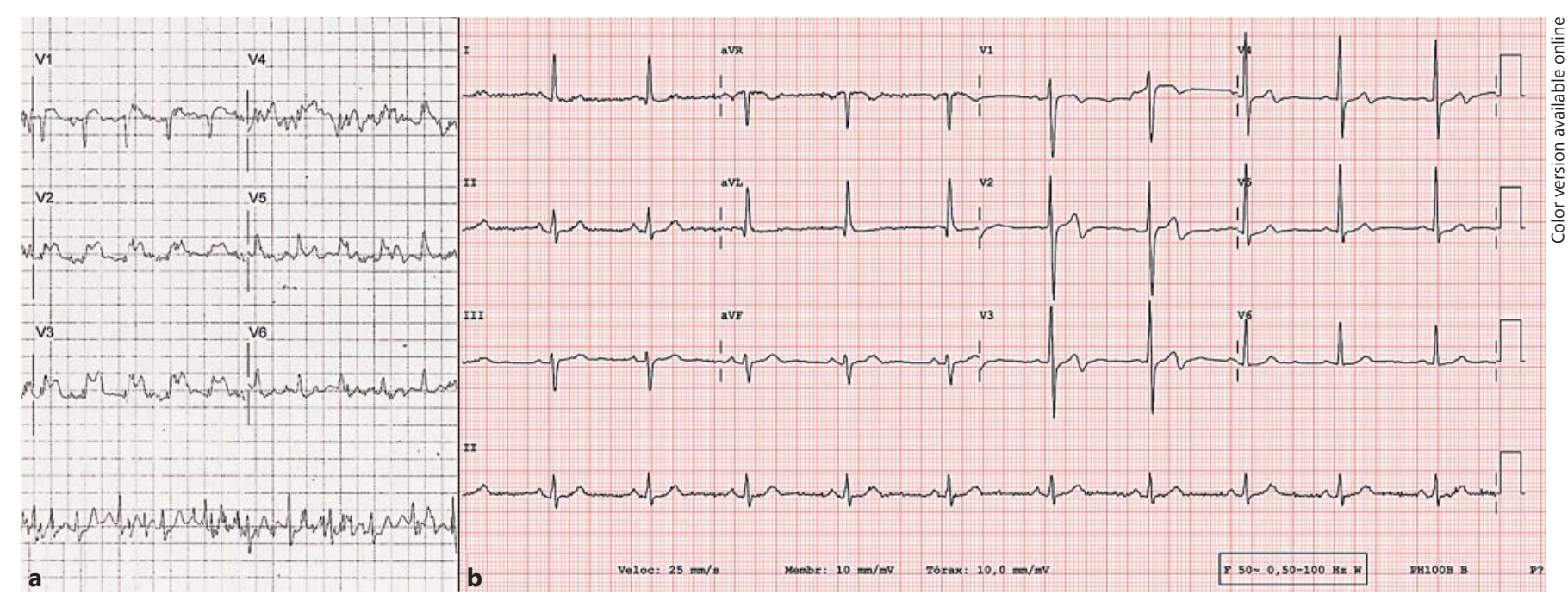

Fig. 2. a Exercise stress test showing an ST-segment elevation of $7 \mathrm{~mm}$ in V2-V4 at 2 min of effort. b Electrocardiogram tracing showing biphasic $\mathrm{T}$ waves in $\mathrm{V} 2-\mathrm{V} 4$.

patient had carotid atherosclerosis and risk factors for coronary heart disease. The echocardiography showing hypokinesis in a region supplied by the LAD further added to our suspicion. The coronariography showed a moderate lesion in the proximal LAD that was not hemodynamically significant, and no treatment intervention was performed. In the original studies, every patient who fulfilled Wellens' criteria had a stenosis in the LAD and in $83 \%$ of these cases the stenosis was proximal. The stenosis could range from $50 \%$ to complete occlusion, and approximately in $17 \%$ of the patients no revascularization treatment was performed [3]. Of these patients, a little over a quarter remained asymptomatic with only medical therapy, which was the case with this patient. An explanation for this might be dissolution of the thrombus formed on top of the stenosis with medical therapy during the time between recognition of the ECG pattern and performance of the coronariography. Another possible explanation might be a coronary spasm, as previously reported by other authors [5], although this hypothesis is less likely since there never was no ST-segment elevation documented during periods of pain. For this reason the patient was not discharged on calcium channel blockers.

The second case is a much more typical presentation of Wellens' syndrome, with characteristic unstable an- 
gina symptoms, biphasic $\mathrm{T}$ waves documented in asymptomatic periods, and a prominent ischemic response in the exercise test, which was very suggestive of a significant proximal LAD stenosis. The biphasic $\mathrm{T}$ waves present at the beginning of the stress test were missed and the decision to proceed with the test could have resulted in an anterior MI [2]. Of the original patient group, only $12 \%$ had cardiac biomarker elevations and these were always minor [3]. This patient had elevation of a biomarker; however, it is expected that with the wider use of highsensitivity cTnI more of these patients will show a positive troponin value. With conservative management, $75 \%$ of these patients will progress to an anterior $\mathrm{MI}$ in 1-23 days [1], and urgent revascularization is key to their treatment.

\section{Conclusion}

The wide spectrum of Wellens' syndrome ranges from a mildly symptomatic patient with a nonhemodynamically significant lesion, who fares well on medical therapy, to a patient with typical symptoms, severity criteria in a stress test, and the need for urgent revascularization to spare a large area of myocardium the risk of an MI. The accurate identification of Wellens' syndrome is extremely important during the management of patients with coronary artery disease since it allows faster aggressive therapy and eliminates potentially dangerous and time consuming tests that do not reduce the risk of developing an anterior MI.

\section{Conflict of Interest}

There is no conflict of interest to report.

\section{References}

Wellens' Syndrome: A Bad Omen
1 De Zwaan C, Bar FW, Wellens HJ: Characteristic electrocardiographic pattern indicating a critical stenosis high in left anterior descending coronary artery in patients admitted because of impending myocardial infarction. Am Heart J 1982;103:730-736.

2 Tandy TK, Bottomy DP, Lewis JG: Wellens' syndrome. Ann Emerg Med 1999;33:347-351.

3 de Zwaan C, Bär FW, Janssen JH, Cheriex EC, Dassen WR, Brugada P, Penn OC, Wellens $\mathrm{HJ}$ : Angiographic and clinical characteristics of patients with unstable angina showing an ECG pattern indicating critical narrowing of the proximal LAD coronary artery. Am Heart J 1989;117:657-665.

4 Rhinehardt J, Brady WJ, Perron AP, Mattu A: Electrocardiographic manifestations of Wellens syndrome. Am J Emerg Med 2002; 20:638-643.

5 Abulaiti A, Aini R, Xu H, Song Z: A special case of Wellens' syndrome. J Cardiovasc Dis Res 2013;4:51-54. 